\title{
GISAXS/GIXRD View of ZnO Films with Hierarchical Structural Elements
}

\author{
M. Lučić Lavčević, ${ }^{1}$ S. Bernstorff, ${ }^{2}$ P. Dubček, ${ }^{3}$ D. Jozić, ${ }^{1}$ \\ I. Jerković, ${ }^{1}$ and Z. Marijanovićc ${ }^{4}$ \\ ${ }^{1}$ Department of Physics, Department of Inorganic Technology, and Department of Chemistry, \\ Faculty of Chemistry and Technology, University of Split, Teslina 10, 21000 Split, Croatia \\ ${ }^{2}$ SAXS beamline, Sincrotrone Trieste, Strada Statale 14, km 163,5, AREA Science Park, 34149 Basovizza, Italy \\ ${ }^{3}$ Division of Materials Physics, Ruđer Bošković Institute, Bijenička 54, 10000 Zagreb, Croatia \\ ${ }^{4}$ Polytechnic "Marko Marulić", Krešimirova 30, 22300 Knin, Croatia
}

Correspondence should be addressed to M. Lučić Lavčević, malula@ktf-split.hr

Received 3 August 2012; Accepted 9 November 2012

Academic Editor: Thomas Thundat

Copyright ( 2012 M. Lučić Lavčević et al. This is an open access article distributed under the Creative Commons Attribution License, which permits unrestricted use, distribution, and reproduction in any medium, provided the original work is properly cited.

\begin{abstract}
$\mathrm{ZnO}$ films constituted of porous sheet-like structures, formed by calcination of precursor, were examined using scanning electron microscopy and simultaneous small-angle scattering and diffraction of the synchrotron-sourced X-rays, under the grazingincidence conditions. The presented analysis enabled insight into the complexity of the film morphology, which revealed substrate sensitivity on the microscopic and nanoscopic length scales. The average size and spatial arrangement of nanoparticles, singlecrystal domains, and the average size and features of nanopores in sheet-like structures were determined for films deposited on glass, polycrystalline $\mathrm{ZnO}$ layer, and silicon.
\end{abstract}

\section{Introduction}

Among the materials with dimensions in the range of microns to nanometers, a variety of $\mathrm{ZnO}$ structures have become the subject of numerous researches [1-3]. Generally, $\mathrm{ZnO}$ is a II-VI semiconductor and its features include good transparency, high electron mobility, and wide bandgap. For the use in various devices, it is typically fabricated in the form of thin film [4-7]. It is well known that functional properties of $\mathrm{ZnO}$ films depend on morphologies. For instance, films with different crystalline orientations exhibit different electronic and optical properties. Due to application requirements, it is therefore necessary to finetune the morphology of the film and characterize it in detail.

In this paper we focus on $\mathrm{ZnO}$ films, with hierarchical structural elements builtin, including sheet-like structures, their constituents-nanoparticles, and the spatial arrangement of sheet-like structures and nanoparticles. The porous architecture of such films makes them suitable for application in optoelectronic and sensing devices, as well as in solar cells [8].

There are several reactions and techniques for generating sheet-like $\mathrm{ZnO}$ structures [9-11]. Among them, the highly selective is the procedure of hydrothermal synthesis and thermal decomposition of a layered precursor of zinc hydroxide carbonate [11]. It was already investigated how the choice of starting chemicals, concentration, temperature, and $\mathrm{pH}$ affect the appearance, size, and arrangement of sheet-like structures in films $[8,11]$. Determination of their morphology was mainly based on electron microscopy (EM), X-ray diffraction (XRD), and measurements based on nitrogen adsorption (BET). Along with characterization of films by common (EM and XRD) techniques, we here present and test the possibilities of a specific method for the analysis of sheet-like structures in $\mathrm{ZnO}$ films, which were formed via a selected procedure on substrates of different types.

The analysis is based on the simultaneous grazing incidence small-angle X-ray scattering and grazing 
incidence X-ray diffraction (GISAXS/GIXRD) measurements, performed by using synchrotron radiation. GISAXS and GIXRD are nondestructive techniques, especially suitable for characterization of thin films and nanosized objects [12-15]. Instead of a localised information of the nanostructure, they provide averages across the irradiated area of the sample. The advantage of simultaneous measurements is based on the complementarity of the two techniques that probe the structural properties on different scales [16]. It is shown that the comparative GISAXS/GIXRD analysis of investigated $\mathrm{ZnO}$ films can provide a notable insight into the complex morphology, which evolves in sheet-like structures upon calcination of the precursor.

The morphological characteristics of analyzed films showed significant substrate sensitivity. Therefore, their comprehensive description on the microscale and the nanoscale may be of applied interest, while combining films with different substrates in functional devices.

\section{Experimental}

2.1. Preparation Route. Three samples of $\mathrm{ZnO}$ films were prepared: sample A on a bare glass substrate, sample B on a $\mathrm{ZnO}$-coated glass substrate, and sample $\mathrm{C}$ on a singlecrystal Si (100) wafer substrate. ZnO coating for sample B was obtained by electron-beam evaporation technique, as a $200 \mathrm{~nm}$ thick polycrystalline layer on the glass substrate.

Films were simultaneously grown on substrates, under the identical condition, following a well-known procedure $[8,11]$. First, template films of $\mathrm{Zn}_{4} \mathrm{CO}_{3}(\mathrm{OH})_{6} \cdot \mathrm{H}_{2} \mathrm{O}$ were deposited from the solution of zinc nitrate hexahydrate and urea in deionized water, in concentrations of $0.05 \mathrm{M}$ and $1.0 \mathrm{M}$, respectively. The $\mathrm{pH}$ value was adjusted to 4 by adding nitric acid to the solution. The substrates were immersed in three bottles with solution and heated in oven at $80^{\circ} \mathrm{C}$ for 6 hours. Finally, the calcination of dried templated films was performed in air at $300^{\circ} \mathrm{C}$, transforming them into $\mathrm{ZnO}$ films.

2.2. Characterization. The insight in general morphologies of the synthesized films was realized in real space, by field emission scanning electron microscopy (FE-SEM Jeol 7000F).

The analysis of nanosized $\mathrm{ZnO}$ particles/crystallites is based on the simultaneous GISAXS/GIXRD measurements. Scattering experiments were performed at the Austrian SAXS beamline at the Synchrotron Elettra (Trieste, Italy) [17]. A photon energy of $8 \mathrm{keV}(\lambda=0.155 \mathrm{~nm})$ was used, and the beam size was $4 \mathrm{~mm} \times 0.150 \mathrm{~mm}$ (horizontal $\times$ vertical). The measurements were performed at a grazing-incident angle, larger than the so-called critical angle for total external reflection, at which X-rays penetrate the sample. The critical angle (around $0,37^{\circ}$ ) was determined experimentally for each film [18]. In the used setup for GISAXS/GIXRD measurements, samples were mounted on a stepping-motorcontrolled tilting stage with a step resolution of $0.001^{\circ}$. At first, the stage (and the sample surface) was aligned horizontally and parallel to the incoming beam within $\pm 0.1^{\circ}$.

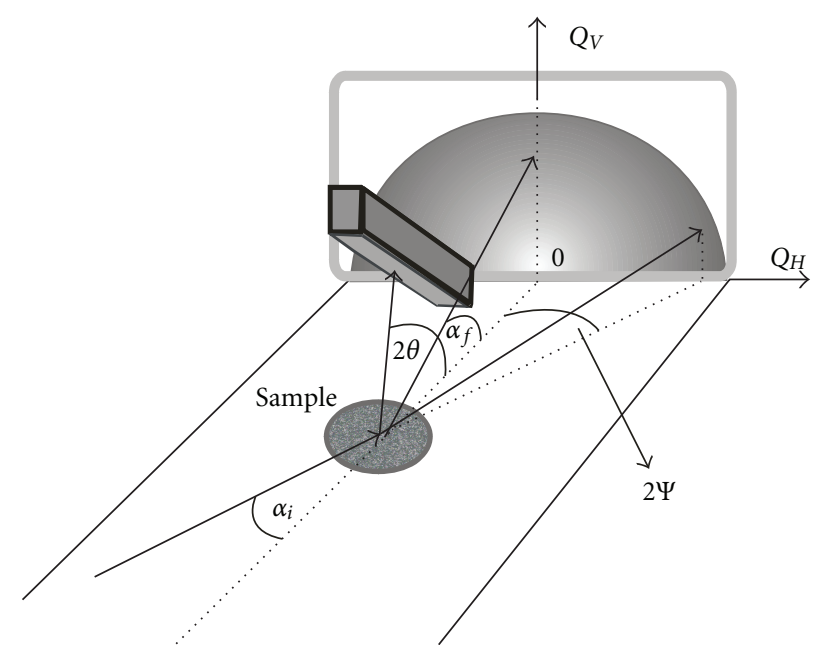

FIGURE 1: The geometry of GISAXS/GIXRD measurements: $\alpha_{i}$ and $\alpha_{f}$ are the incident and the exit angle of the X-ray beam, respectively; $\psi$ is the scattering in-plane angle; $Q_{H}$ and $Q_{V}$ are the horizontal and the vertical scattering vector, respectively; $2 \theta$ is the diffraction angle.

The configuration for GISAXS and GIXRD measurement is illustrated in Figure 1.

The GISAXS patterns were recorded by a two dimensional charge coupled device (2D CCD) detector, containing $1024 \times 1024$ pixels and positioned perpendicular to the incident beam. A thin Al strip was placed in front of the detector to avoid its overflow.

Two main directions, horizontal (parallel to the sample plane) and vertical (perpendicular to the sample plane), are represented by the position vectors in reciprocal space, the scattering vectors $Q_{H}$ and $Q_{V}$, respectively. Their moduli are defined by the wavelength $\lambda$, the incidence and exit angles $\alpha_{i}$ and $\alpha_{f}$, and the in-plane scattering angle $2 \psi$ of X-rays as

$$
Q_{H}=2 \pi \lambda^{-1} \cos \alpha_{f} \sin \psi, \quad Q_{V}=2 \pi \lambda^{-1}\left(\sin \alpha_{i}+\sin \alpha_{f}\right) .
$$

The GIXRD measurements were performed simultaneously in the reflectivity plain, using a position sensitive detector (PSD), which covered an angular range $(2 \theta)$ from $20^{\circ}$ to $50^{\circ}$. Phase identification was realized matching diffracted peaks with PDF cards.

The GISAXS/GIXRD patterns were corrected for readout noise and detector response.

Conventional XRD measurements (in Bragg-Brentano geometry) were performed on samples using Philips X'pert diffractometer.

\section{Results and Discussion}

3.1. FESEM View of the General Microscopic and Nanoscopic Morphology. The general morphologies of the samples A, B, and C, analyzed by FE-SEM, are shown in Figures 2(a), 2(b), and $2(\mathrm{c})$. The FE-SEM images clearly exhibit that substrates 


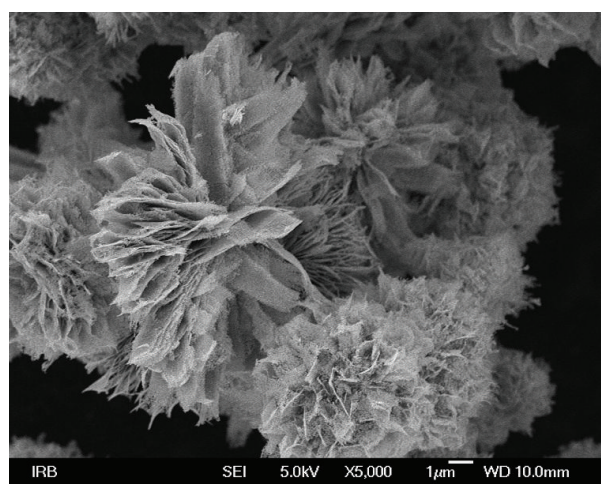

(a)

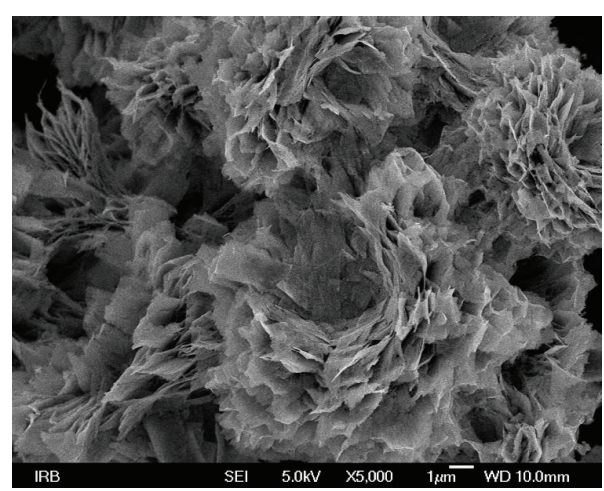

(b)

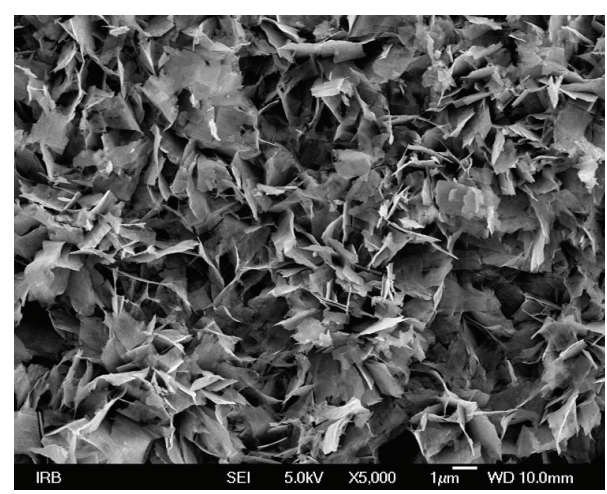

(c)

FIgUre 2: FE-SEM pictures of sample A (a), sample B (b), and sample C (c).

are covered by nanostructures, which generally possess sheetlike morphologies. Sheets are about few micrometers in dimension and about $60 \mathrm{~nm}$ in thickness. The nanosized thickness classifies them as nanosheets. However, there are differences in both microscopic and nanoscopic morphology between samples A, B, and C obtained on amorphous (glass), polycrystalline $(\mathrm{ZnO})$, and single crystal ( $\mathrm{Si}$ ) substrates, respectively.

In samples A and B nanosheets are self-organized as nanopetals, forming flower-like structures. In case of sample A, such flowers are about $5 \mu \mathrm{m}$ in dimension and show diversity in shape: mostly they are bush-like with randomly oriented petals or sprouts, assembled in a radiative way; otherwise, they adopt a shape of flowers in full blow. In sample B, flowers are bigger, about $8 \mu \mathrm{m}$ in dimension; they have mostly regular form of rose-like flowers, with petals forming hollow central parts. Because of the specific spatial arrangement of nanopetals, the films are porous systems. Furthermore, a nanoscopic FE-SEM view reveals the inner porosity of the petals themselves: in both samples they are formed as networks of more or less agglomerated nanosized particles and nanopores (Figures 3(a) and 3(b)).

In case of sample $C$, sheet-like nanostructures are not self-organized in flowers. They appear as individual truncated nanoleaves. Also, they are composed in a different manner, their nanoscopic FE-SEM image (Figure 3(c)) exhibits a less porous structure.
Evidently, the analysis by FE-SEM shows that the morphology of $\mathrm{ZnO}$ films is substrate dependent.

The microdimensional constituents (flowers and leaves) of $\mathrm{ZnO}$ films, shown in Figure 2, were obtained via a two-step synthesis, specified in Section 2. In the first step, template films consisting of microdimensional structures of $\mathrm{Zn}_{4} \mathrm{CO}_{3}(\mathrm{OH})_{6} \cdot \mathrm{H}_{2} \mathrm{O}$ were formed. In the second step, during the calcination, template films were transformed into $\mathrm{ZnO}$ films. According to the literature, final films preserve almost identical microscopic morphology (size and shape of flowers and leaves) as those obtained in the first step. Therefore $\mathrm{Zn}_{4} \mathrm{CO}_{3}(\mathrm{OH})_{6} \cdot \mathrm{H}_{2} \mathrm{O}$ acts as a self-template precursor and the final film morphology reflects the manner of growth of its layered structure. Under this assumption, we can conclude that the observed substrate dependence of the microscopic morphology of the films originates from the mechanism of the nucleation and growth of the $\mathrm{Zn}_{4} \mathrm{CO}_{3}(\mathrm{OH})_{6} \cdot \mathrm{H}_{2} \mathrm{O}$ on different substrates. The precursor nuclei are formed on the substrate surface by heterogeneous nucleation. There is no growth hindrance in the azimuthal direction until the growing sheets come into contact with each other. Then the self-assembled microstructures of the precursor nanosheets are generally made, like flowers in samples A and B. From the thermodynamics point of view, nanosheets tend to aggregate to decrease the surface energy by reducing exposed areas; besides, the interaction between the deposit material and the substrate surface must be taken into account. 


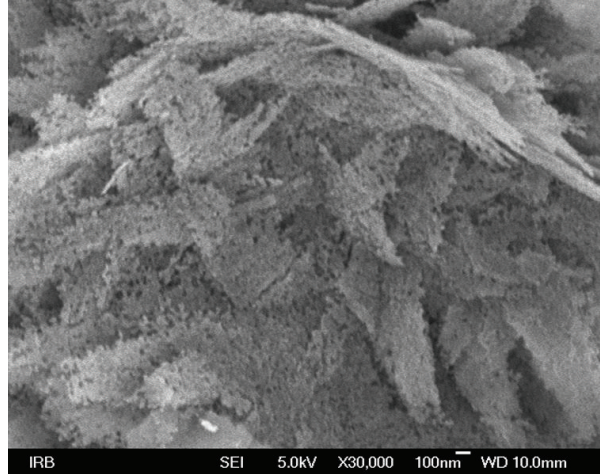

(a)

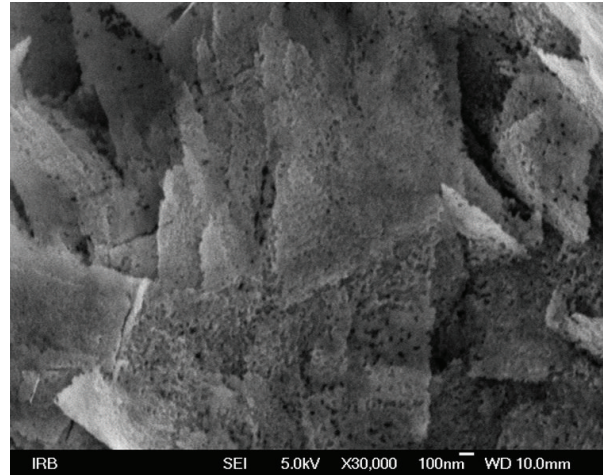

(b)

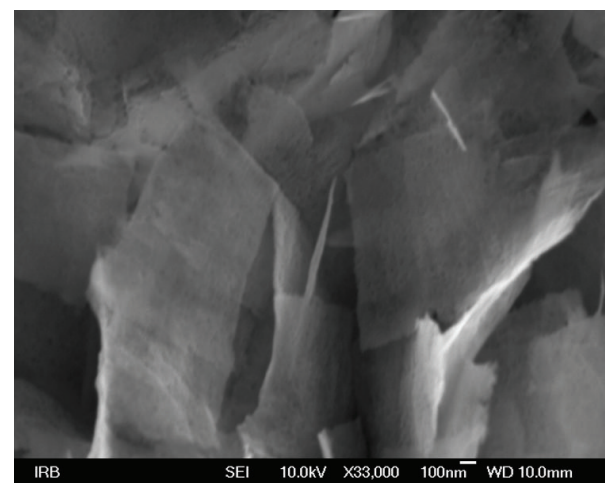

(c)

Figure 3: FE-SEM pictures—nanoscopic view—of sample A (a), sample B (b), and sample C (c).

The number and type of nucleation sites basically depend on the substrate characteristics, such as roughness, chemical composition, and crystallographic structure. Furthermore, the hydrophilic/hydrophobic interactions between the growing sheets and the substrate can affect the manner of the film growth. Although the assembling of nanosheets in flowerlike structures takes place in samples A and B, forming the same general morphology in both samples, flowers grown on polycrystalline $\mathrm{ZnO}$ substrate have more regular shape and sheet distribution than those grown on amorphous glass surface. One of the possible reasons may be a certain degree of similarity between the structure of the substrate and the precursor. Furthermore, the tendency of petals in sample A to direct themselves upwards, rather than to spread on the substrate, is probably caused by the difference in the hydrophobicity of the surface of $\mathrm{Zn}_{4} \mathrm{CO}_{3}(\mathrm{OH})_{6} \cdot \mathrm{H}_{2} \mathrm{O}$ and the surface of $\mathrm{ZnO}$ nanostructured substrate. Finally, the absence of the self-assembling of sheets in sample C can also be tentatively ascribed to hydrophobic interactions and geometric effects between the precursor and the singlecrystal silicon surface.

Differences between the as-prepared precursor and the final $\mathrm{ZnO}$ film are mostly related to the nanoscopic morphology of their structures and are caused by the formation of pores during the heating process.

The formation of the porous network in a petal takes place during the conversion of the $\mathrm{Zn}_{4} \mathrm{CO}_{3}(\mathrm{OH})_{6}$ precursor to $\mathrm{ZnO}$ at $300^{\circ} \mathrm{C}$, according to the following chemical reaction:

$$
\mathrm{Zn}_{4} \mathrm{CO}_{3}(\mathrm{OH})_{6} \mathrm{H}_{2} \mathrm{O}+\mathrm{O}_{2} \longrightarrow 4 \mathrm{ZnO}+4 \mathrm{H}_{2} \mathrm{O}+\mathrm{CO}_{2}
$$

During the thermal process, besides small $\mathrm{ZnO}$ units, vacancies are formed that balance the released molecules $\mathrm{H}_{2} \mathrm{O}$ and $\mathrm{CO}_{2}$. The nanosized pores in the sheets might come from the effect, in which the vacancies formed in the initial period condense to form voids. Additionally, after the initial period and with increasing temperature, $\mathrm{ZnO}$ units assemble into nanoparticles.

In summary, the FE-SEM view suggests that the morphology of films exhibits substrate sensitivity and can be considered in the frame of four hierarchical elements: single sheet, single nanoparticle, spatial arrangement of the sheets, and spatial arrangement of nanoparticles.

GIXRD and GISAXS enable an insight in this complex morphology on the nanoscopic level.

3.2. GIXRD View of the Nanoscopic Morphology and Crystallographic Structure. From GIXRD patterns, shown in Figures 4(a), 4(b), and 4(c), the crystallographic structure of the samples was examined. The recorded diffraction peaks match with crystalline hexagonal wurtzite-type structure and correspond to (100), (002), and (101) planes of $\mathrm{ZnO}$, respectively. 


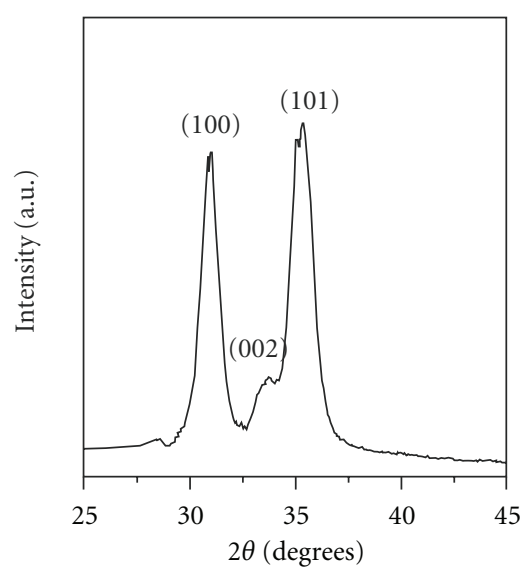

(a)

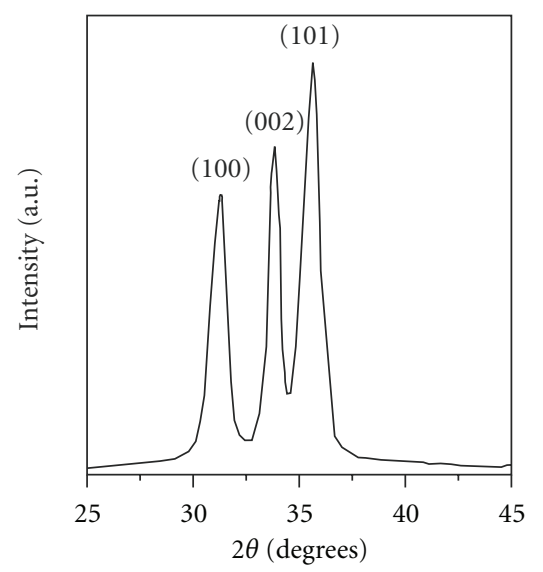

(b)

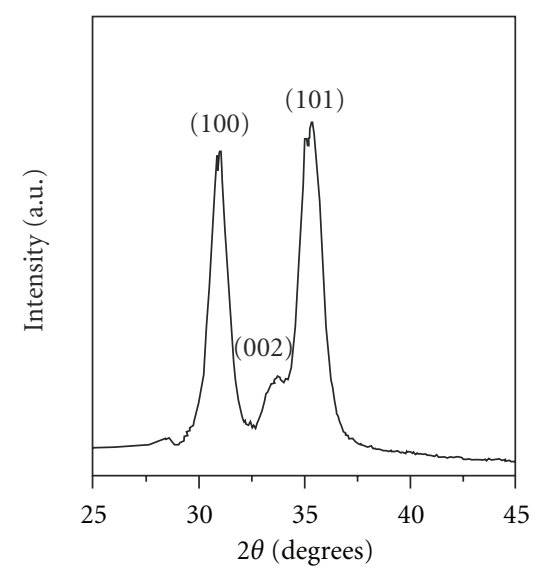

(c)

FIgURE 4: GIXRD patterns of sample A (a), sample B (b), and sample C (c).

The relative intensities of the peaks in the GIXRD patterns differ among the samples, indicating different degrees of crystallographic orientation in films. GIXRD can not be used for a straightforward interpretation of the intensity scans considering the preferred orientations: as the incident angle is fixed, the scan normal varies with the diffraction angle. Therefore, each diffraction peak in a grazing incidence pattern corresponds to a different orientation of the reflecting planes. Since the geometry of measurements is not symmetrical, some reflections can be hindered. For an accurate estimate of the texture, conventional XRD measurements on samples (shown later in text) are needed. However, some assumptions about orientation can be made using GIXRD. As shown in Figure 4, all dominant peaks appear in the GIXRD patterns of samples A and B, indicating that the single crystal domains in these samples are either randomly oriented or eventually weakly ordered. On the other hand, the pattern of sample $\mathrm{C}$ exhibits a dominant contribution of the (100) and (101) peaks and a barely resoluble (002) peak. Such pattern, recorded in the grazing incidence mode, suggests that a significant portion of single crystal domains in this sample is oriented with their c-axis parallel to the film surface, since the tilt between the (100) plane and the scan normal is close to $90^{\circ}$ for the given grazing incident angle.

The broadening of diffraction peaks reveals that the size of single crystal domains is in the nanometer range, that is, they are nanocrystals. The average size was calculated from the profile width at half maximum height of the given peaks, using the following Scherrer equation [19]:

$$
L(h k l)=\frac{K \lambda}{\beta \cos \theta},
$$

where $L(h k l)$ is the average nanocrystal size (which may be smaller or equal to the particle size) estimated from the ( $h k l)$ reflection, $\lambda(=0.1554 \mathrm{~nm})$ is the wavelength of synchrotron sourced $\mathrm{X}$-rays, $\beta$ is the broadening of diffraction line measured at half its maximum intensity in radians, and $\theta$ is the angular peak position. According to the widespread practice, a value of one was retained for Scherrer constant $K$. Generally, the Scherrer constant depends on the crystallite shape and on the size distribution. However, it is not a bad approximation in this case, if, for each $(h k l)$ reflection, the crystallite size $L$ is interpreted as an average crystal dimension perpendicular to the corresponding reflecting plane. The average nanocrystal sizes in samples A, B, and $\mathrm{C}$ were calculated from the dominant peaks of $\mathrm{ZnO}$. The instrumental broadening of $0.2^{\circ}$ was incorporated in the size calculations.

The estimated sizes, much smaller than any dimension of the sheets, complement the microscopic picture showing that sheets exhibit polycrystalline structure.

The average nanocrystal sizes in samples A and B are similar, with the exception of one enhanced size, estimated from the (002) peak in the pattern of sample B. The average crystallite sizes in sample $\mathrm{C}$ are slightly lower and can be properly estimated only for the (100) and (101) reflections. Similar nanocrystal sizes, estimated from different peaks of the sample A, suggest that it consists of isotropically shaped or randomly oriented nanocrystals. The (002) peak of sample $B$, sharper than others and stronger than that of standard pattern, indicates that nanocrystals have anisotropic shape, elongated in the direction of the c-axis.

According to the FE-SEM pictures, the average size of single nanoparticles, agglomerated in petals, correlate well with the estimated average size of nanocrystals. We can conclude that most of the single nanoparticles in petals are single crystal domains. This conclusion does not apply to particles forming leaves, as their size cannot be properly estimated by FE-SEM.

3.3. GISAXS View of the Nanoscopic Morphology. The FESEM analysis allow us to consider petals and leaves in $\mathrm{ZnO}$ films as two-phased systems, composed of nanosized particles and pores. As the SAXS signal arises from the difference in electron density between the two phases, it is suitable for the analysis of the porous samples. Their 2D GISAXS patterns, given in Figure 5, represent the maps of 


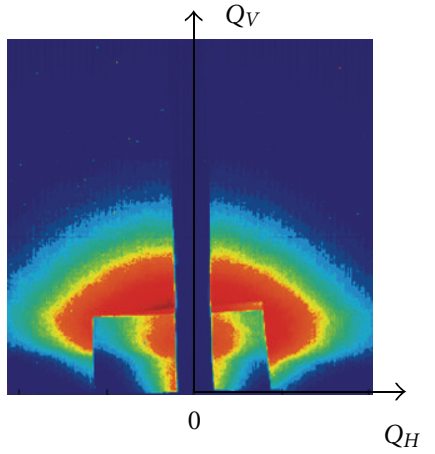

(a)

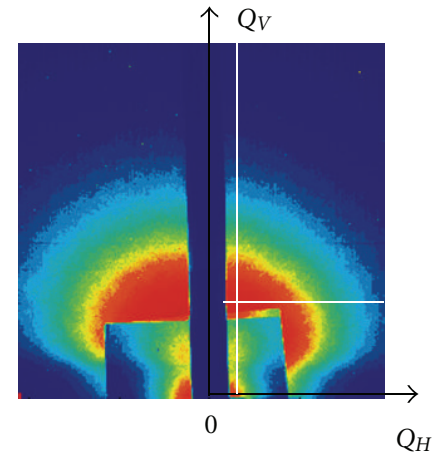

(b)

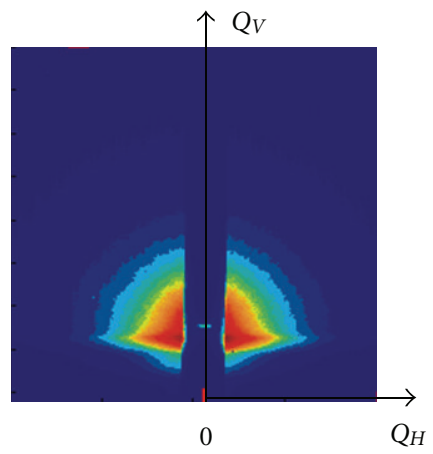

(c)

Figure 5: 2D GISAXS patterns of sample A (a), sample B (b), and sample C (c).

scattered intensities in the given in-plane and out-of-plane angular scattering ranges. The maximum of the scattered intensity is in the direction of the specular plane $\left(Q_{H}=0\right)$, which is normal to the surface of the sample. This scattered intensity is partly reduced by a thin $\mathrm{Al}$ absorber (vertical strips in the patterns are depleted intensities). The lower part of the scattering is missing because of the absorption in the sample. For exit angles equal to incident critical angle $\left(\alpha_{f}=\alpha_{c}\right)$, there is an enhancement in the scattering intensity due to the refraction effects, which is also known as Yoneda peak. The presence of small angle X-ray scattering in patterns indicates the existence of nanosized heterogeneities in electron density in all samples. 2D GISAXS patterns allow determination of dimensions of the scattering objects along horizontal and vertical direction, represented by the scattering vectors $Q_{H}$ and $Q_{V}$, respectively. The method of interpreting scattering data is based on the analysis of the intensity cuts, which show the dependence of the intensity, $I$, on the scattering vector, $Q_{i}\left(Q_{H}\right.$ or $\left.Q_{V}\right)$. The intensity cuts, that is, one dimensional scattering curves, taken from Figure 5 at fixed values of horizontal and vertical scattering vectors (relatively close to the Yoneda peak and to the specular plane), were used to calculate the so-called Guinier radii, $R_{G V}$ and $R_{G H}$, which give information about the average size in given directions.

The white strips indicate where the intensity cut shown in Figures 6 and 7 was taken.

In the Guinier approximation [20], scattering in the small Q-region depends only on the overall size of the object and not on its shape, so the scattered intensity $I(Q)$ can be expressed as

$$
I(Q)=C_{G} \exp \left(-\frac{R_{G}^{2} Q^{2}}{3}\right) .
$$

The Guinier radius $R_{G}$ is the average radius of gyration of a dilute set of randomly oriented scattering centers. When this condition is not fulfilled, it still can be calculated as a rough approximation. In order to determine the gyration radii, we used the so-called Guinier graph: we plotted the intensity cuts in the $\operatorname{Ln} I$ versus $Q_{i}^{2}$ presentation and fitted each of them by a line for very small scattering vectors. As example, the fitting of a vertical and a horizontal cut is

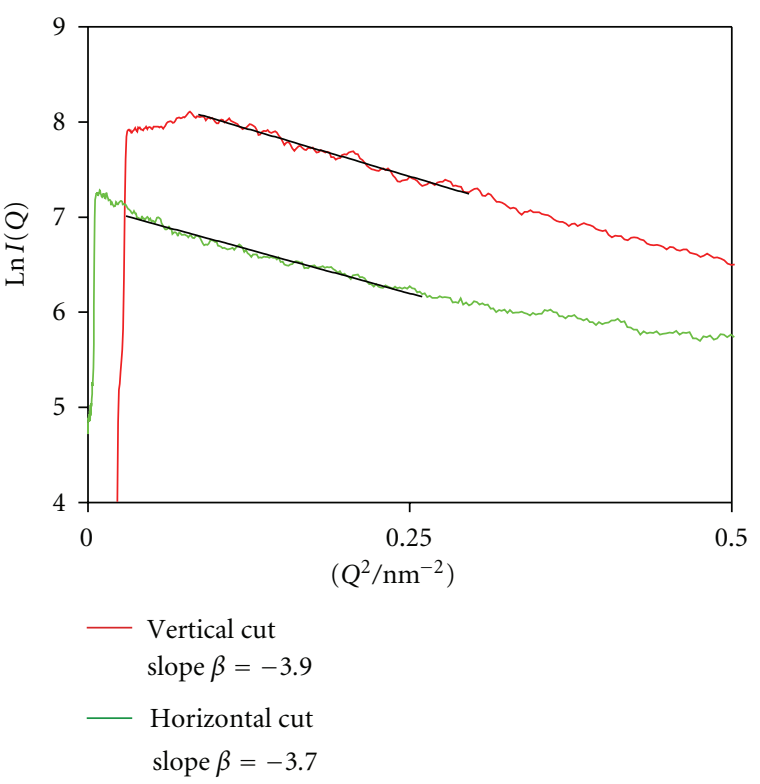

FIGURE 6: Scattering curves of sample B, extracted from Figure 5(b) as vertical cut at a constant value of the horizontal wave vector (red) and horizontal cut at a constant value of the vertical wave vector (green), shown in the presentation suitable for determination of the average gyration radius. $\beta$ is the slope of the fitting lines (black).

represented in Figure 6 for sample B. The two white strips in Figure 5(b) indicate where the 1D cut shown in Figure 6 was taken.

For a slope $\beta$ of the fitting line, the corresponding Guinier radius is calculated as

$$
R_{G}=(-3 \beta)^{1 / 2} .
$$

The determined gyration radii $R_{G H}$ and $R_{G V}$ for the samples $\mathrm{A}, \mathrm{B}$, and $\mathrm{C}$ are shown in Table 2.

The Guinier radii for sample B obtained from a horizontal and a vertical intensity scan have similar values, which suggest that scattering objects are either spherical or anisotropic forms with random orientation. The average 
TABle 1: The GIXRD data of the $\mathrm{ZnO}$ thin films: crystallite sizes, $L(h k l)$, estimated from (100), (002), and (101) reflections.

\begin{tabular}{lccc}
\hline Sample & $L(100) / \mathrm{nm}$ & $L(002) / \mathrm{nm}$ & $L(101) / \mathrm{nm}$ \\
\hline A & 13 & 14 & 12 \\
B & 14 & 17 & 13 \\
C & 12 & $/$ & 11 \\
\hline
\end{tabular}

TABle 2: The Guinier radii, $R_{G H}$, and $R_{G V}$ for samples $\mathrm{A}, \mathrm{B}$, and C, calculated from vertical and horizontal intensity cuts.

\begin{tabular}{lcc}
\hline Sample & $R_{G H} / \mathrm{nm}$ & $R_{G V} / \mathrm{nm}$ \\
\hline A & 3.1 & 3.5 \\
B & 3.3 & 3.4 \\
C & 4.2 & 2.2 \\
\hline
\end{tabular}

diameter, $D$, for a sphere can be calculated from the following average gyration radius:

$$
D=2 R_{G} \sqrt{\frac{5}{3}} .
$$

The resulting average diameter of $8.8 \mathrm{~nm}$ does not correspond to the average crystallite size in this sample (Table 1). As crystallites can not be bigger than particles, this result reveals that scattering objects in sample B are not particles, but nanosized pores inside the petals. The same conclusion applies to sample A, although in this sample the radii of gyration differ in the two directions, suggesting the slight anisotropy of pores. Anisotropy in SAXS results from more or less oriented scattering objects. Although Guinier approximation gives only the radius as a measure of size, it is also partly sensitive to oriented flat or elongated scattering objects. The analysis of vertical and horizontal scans of sample $C$ showed the significant difference in the corresponding average gyration radii $\left(R_{G H} / R_{G V}=1.9\right)$. Therefore, the anisotropy of scattering objects (also evident from the shape of the 2D GISAXS pattern in Figure 5(c)) is assumed. It can be related to the anisotropy in crystallite orientations of this sample, shown by the GIWAXS. The size of scattering objects, suggested by the value of the gyration radii, is comparable to the size of crystallites in this sample. However, considering the more dense structure of leaves in comparison to petals, the scattering can still be ascribed to pores.

Besides the Guinier graph, the so-called Porod graph can be exploited for the further analysis of scattering. In the Porod approximation for a two-phase system [21], the scattering radiation in the high Q-region is probing the local structure. For this analysis, the intensity cuts from Figure 5 were plotted in the $\operatorname{Ln} I$ versus $\operatorname{Ln} Q_{i}$ presentation and fitted by a line for high scattering vectors. The slope $n$ of the fitting line yields information about the so-called "fractal dimension" of the scattering objects. As example, the Porod graph obtained from the intensity cut of the sample B (Figure 5(b)) is shown in Figure 7.

The Porod slopes estimated for samples A, B, and C, as the two-phased systems of particles and pores, are -3.1 ,

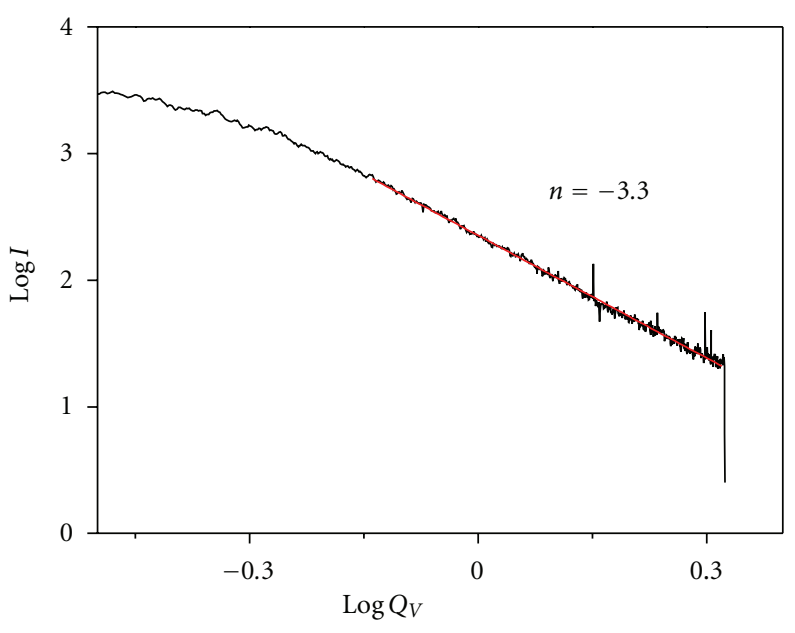

FIGURE 7: The Porod graph, obtained from the vertical intensity cut of sample B (shown in Figure 5(b)); nis the slope of the fitting line (red).

-3.3 , and -4 , respectively. The slopes determined for samples $A$ and $B$ represent rough interfaces between particles and pores, while slope -4 indicates a smooth interface in sample C. Again, the exceptional behavior of morphological characteristics of this sample is confirmed. Leaves, as its structure elements, exhibit not only the lack of self-organizing, but also a different inner structure: while evolving in leaves, the constituent $\mathrm{ZnO}$ units are stacked into anisotropic forms with smooth surfaces, arranged more densely than those in petals. As compared to the other two samples, the more pronounced Yoneda peak in the scattering pattern of sample $\mathrm{C}$ additionally confirms the more smoothed surface.

In order to verify the GISAXS/GIXRD view of samples and enhance the information on their structural properties, the samples were finally investigated by XRD in BraggBrentano geometry.

3.4. Conventional XRD Measurements. Conventional XRD measurements enable determination of reflections from the crystal planes which are parallel to the sample surface. XRD patterns of samples A, B, and C, shown in Figure 8, were recorded in a wider angular range in comparison to that used in the GIXRD measurements. Therefore, two additional peaks are visible, which correspond to (102) and (110) reflecting planes. The contribution of the amorphous glass substrate is evident for samples A and B.

Average nanocrystal sizes, $L(h k l)$, estimated using (3) from the dominant (100), (002), and (101) reflections, are given in Table 3. Crystallite sizes obtained from conventional XRD correlate well with those obtained from GIXRD. Taking into account that XRD data refer to the entire sample, while those of GIXRD describe only its upper part, we can conclude that the petals and leaves have uniform inner structure on the whole.

As in the case of GIXRD patterns, the relative intensities of the peaks in the XRD patterns differ among the samples. However, unlike GIXRD, conventional XRD can be used 


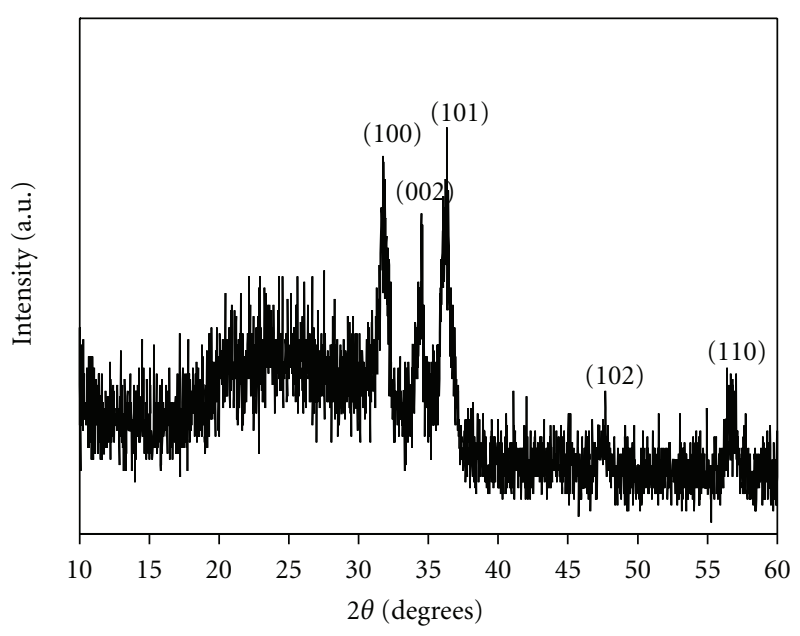

(a)

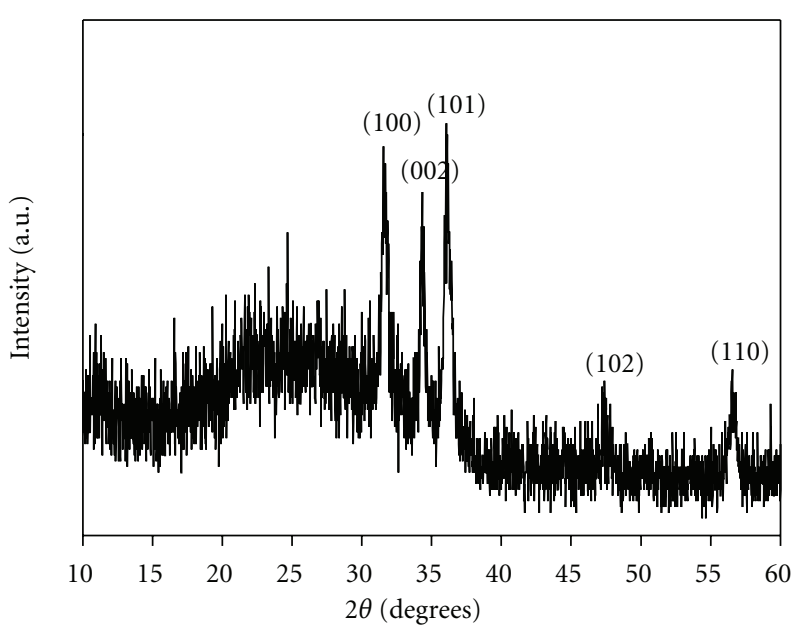

(b)

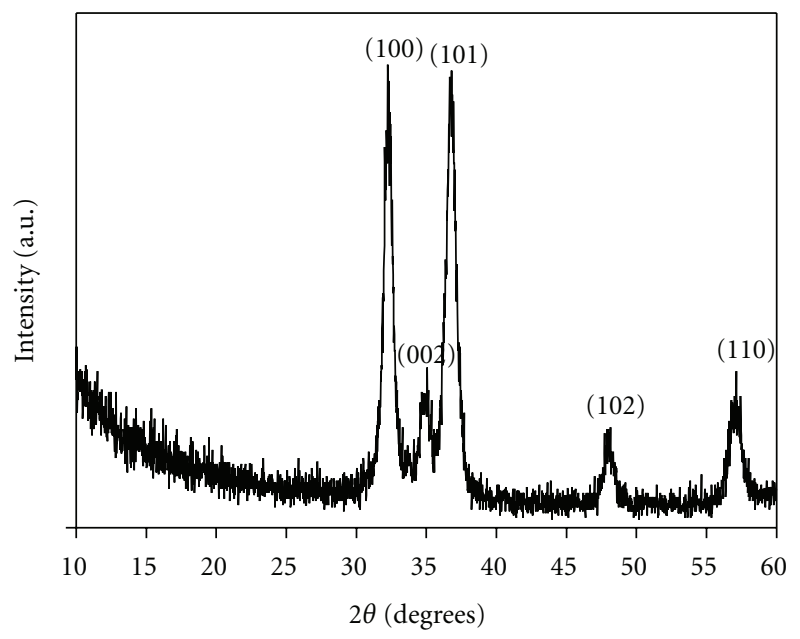

(c)

Figure 8: XRD patterns of sample A (a), sample B (b), and sample C (c).

TABle 3: Average nanocrystal sizes, $L(h k l)$, estimated from (100), (002), and (101) reflections, from conventional XRD measurements.

\begin{tabular}{lccc}
\hline Sample & $L(100) / \mathrm{nm}$ & $L(002) / \mathrm{nm}$ & $L(101) / \mathrm{nm}$ \\
\hline A & 12 & 12 & 11 \\
B & 14 & 18 & 14 \\
C & 13 & $/$ & 11 \\
\hline
\end{tabular}

for a straightforward interpretation of the intensity scans considering the preferred orientations. The estimation of the film texture is generally based on the comparison of the orientation distribution of single crystal domains in film with entirely random orientation of domains in a powder sample, taken as standard. The texture of the particular plane can be presented by the texture coefficient [22], deviation of which from unity implies the preferential crystallite orientation. So, the texture coefficient $T(h k l)$ is defined as

$$
T(h k l)=\frac{I(h k l) / I_{0}(h k l)}{n^{-1} \sum_{n}\left(I(h k l) / I_{0}(h k l)\right)},
$$

TABle 4: Texture coefficients, $T(h k l)$, estimated from (100), (002), (101), (102), and (110) reflections, from conventional XRD measurements.

\begin{tabular}{lccccc}
\hline Sample & $T(100)$ & $T(002)$ & $T(101)$ & $T(102)$ & $T(110)$ \\
\hline A & 1.1 & 1.2 & 0.9 & 1.1 & 0.8 \\
B & 1.0 & 1.5 & 0.7 & 1.0 & 0.8 \\
C & 1.8 & 0.6 & 0.9 & 0.7 & 1.0 \\
\hline
\end{tabular}

where $I(h k l)$ is the measured relative intensity of a plane $(h k l), I_{0}(h k l)$ is the standard intensity of the plane $(h k l)$ taken from the JCPDS data, and $n$ is the number of diffraction peaks. In the present analysis, $n=5$, because five directions are involved (100), (002), (101), (102), and (110). A sample with randomly oriented crystallite presents $T(h k l)=1$, while the larger this value, the larger the abundance of domains oriented at the $(h k l)$ direction. The estimated texture coefficients (shown in Table 4) fit into the image of nanoscopic morphology, formed by this point. 
The values of the texture coefficient confirm the difference in the spatial orientation of nanocrystals between samples, indicated by GIXRD and probably caused by the substrate-induced difference on the microscale: the nanocrystals in petals of flowers on glass are randomly oriented, and those in petals of rose-like flowers on polycrystalline $\mathrm{ZnO}$ exhibit a weak (002) texture. Further, the portion of nanocrystals arranged with their c-axis perpendicular to the substrate is relatively small in leaves grown on silicon. A relatively pronounced (110) reflection in the XRD pattern of this sample confirms its previously presumed texture: besides a number of nanocrystals with c-axis tilted with respect to the substrate, which are partly represented by the (101) reflection, most of nanocrystals seem to be arranged with c-axis parallel to the sample surface. Such arrangement of nanocrystals is common to both orientations (100) and (110).

\section{Conclusion}

In summary, we generated nanostructured $\mathrm{ZnO}$ films with hierarchical structural elements, via hydrothermal synthesis and thermal decomposition of the zinc hydroxide carbonate as precursor, using different substrates. The FE-SEM measurements showed that the nature of substrate has a strong influence on the hierarchical elements (nanosheets and their spatial arrangement) on the microscale. By the combined GISAXS/GIXRD method of analysis, it was elucidated how this substrate sensitivity reflects on the morphology on the nanoscale. The average size and shape of the constituents of nanosheets, nanocrystals/nanoparticles, and nanopores, as well as the nature of the inner surface of the sheets, were estimated for samples on different substrates. The development of different crystallographic orientations of nanocrystals in samples, indicated by GIXRD, was confirmed and defined in detail by XRD. It was found that their orientation is probably ruled by the spatial arrangement of sheets (i.e., by the hierarchical characteristics on the microscale).

However, the texture of sample B, which exhibits a weak out-of-plane ordering related to increased number of nanocrystals with their c-axis perpendicular to the substrate, should be additionally commented. Namely, the substrate in this sample is a $200 \mathrm{~nm}$ thick polycrystalline layer of $\mathrm{ZnO}$, with single crystal domains of average size of $20 \mathrm{~nm}$. The conventional XRD of this layer shows hardly resoluble $\mathrm{ZnO}$ peaks because of the glass absorption, while its GIXRD pattern correlate with that of sample B. This indicates that orientation of nanocrystals may not depend solely on the spatial arrangement of the sheets, but is partly conditioned by the substrate. Further investigations of films deposited on various textured layers should clarify whether the substrate affects the mutual arrangement of nanocrystals/nanoparticles in sheets.

In conclusion, the combined GISAXS/GIXRD method was successful in estimation of the substrate-induced changes in morphology on the nanoscale. Along with FE-SEM, this method allows a comprehensive assessment of the morphological characteristics, which define the properties of film in application. The determined film characteristics can be tuned to be useful in dye-sensitized solar cells (for the enhancement of dye absorption, electrolyte distribution, and electrical pathways via nanoporosity, large surface area, and orientation of nanocrystals), in sensing devices (for the enhanced gas sensing through the enlargement of density in vacancies of the porous sheets) and as catalysts (for the enhancement of activity due to good crystallinity and large surface area).

\section{Acknowledgment}

The results shown in this work are obtained in the frame of the scientific project "Nanostructures of metal oxides and metals: morphology and properties," supported by the Ministry of Science, Education and Sports of Croatia.

\section{References}

[1] L. Kumari, W. Z. Li, C. H. Vannoy, R. M. Leblanc, and D. Z. Wang, "Zinc oxide micro- and nanoparticles: synthesis, structure and optical properties," Materials Research Bulletin, vol. 45, no. 2, pp. 190-196, 2010.

[2] J. Wu and D. Xue, "Progress of science and technology of $\mathrm{ZnO}$ as advanced material," Science of Advanced Materials, vol. 3, no. 2, pp. 127-149, 2011.

[3] S. Musić, S. Popović, M. Maljković, and D. Dragčević, "Influence of synthesis procedure on the formation and properties of zinc oxide," Journal of Alloys and Compounds, vol. 347, no. 1-2, pp. 324-332, 2002.

[4] S. Singh, P. Thiyagarajan, K. Mohan Kant et al., "Structure, microstructure and physical properties of $\mathrm{ZnO}$ based materials in various forms: bulk, thin film and nano," Journal of Physics D, vol. 40, no. 20, pp. 6312-6327, 2007.

[5] L. Zeng, S. Dai, W. Xu, and K. Wang, "Dye-sensitized solar cells based on ZnO films," Plasma Science and Technology, vol. 8, no. 2, pp. 172-175, 2006.

[6] K. Keis, J. Lindgren, S. E. Lindquist, and A. Hagfeldt, "Studies of the adsorption process of Ru complexes in nanoporous $\mathrm{ZnO}$ electrodes," Langmuir, vol. 16, no. 10, pp. 4688-4694, 2000.

[7] U. Ozgur, Y. I. Alivov, C. Liu et al., "A comprehensive review of $\mathrm{ZnO}$ materials and devices," Journal of Applied Physics, vol. 98, no. 4, Article ID 041301, 103 pages, 2005.

[8] K. Kakiuchi, M. Saito, and S. Fujihara, "Fabrication of $\mathrm{ZnO}$ films consisting of densely accumulated mesoporous nanosheets and their dye-sensitized solar cell performance," Thin Solid Films, vol. 516, no. 8, pp. 2026-2030, 2008.

[9] A. Pan, R. Yu, S. Xie, Z. Zhang, C. Jin, and B. Zou, "ZnO flowers made up of thin nanosheets and their optical properties," Journal of Crystal Growth, vol. 282, no. 1-2, pp. 165-172, 2005.

[10] A. E. Suliman, Y. Tang, and L. Xu, "Preparation of $\mathrm{ZnO}$ nanoparticles and nanosheets and their application to dyesensitized solar cells," Solar Energy Materials and Solar Cells, vol. 91, no. 18, pp. 1658-1662, 2007.

[11] K. Kakiuchi, E. Hosono, T. Kimura, H. Imai, and S. Fujihara, "Fabrication of mesoporous $\mathrm{ZnO}$ nanosheets from precursor templates grown in aqueous solutions," Journal of Sol-Gel Science and Technology, vol. 39, no. 1, pp. 63-72, 2006.

[12] J. R. Levine, J. B. Cohen, Y. W. Chung, and P. Georgopoulos, "Grazing-incidence small-angle X-ray scattering: new tool for 
studying thin film growth," Journal of Applied Crystallography, vol. 22, p. 528, 1989.

[13] M. L. Lavčević, P. Dubček, Z. Crnjak Orel, and A. Turković, "GISAXS view of vanadium/cerium oxide thin films and influence of lithium intercalation," Journal of Chemical Information and Modeling, vol. 45, no. 6, pp. 1553-1557, 2005.

[14] M. Lučić Lavčević, A. Turković, P. Dubček, Z. Crnjak Orel, B. Orel, and S. Bernstorff, "GISAXS view of induced morphological changes in of nanostructured CeVO4 thin films," Journal of Nanomaterials, vol. 2011, Article ID 303808, 7 pages, 2011.

[15] M. Lučić Lavčević, P. Dubček, A. Turković, Z. CrnjakOrel, and S. Bernstorff, "Nanostructural depth profile of vanadium/cerium oxide film as a host for lithium ions," Solar Energy Materials and Solar Cells, vol. 91, no. 7, pp. 616-620, 2007.

[16] M. L. Lavčević and A. Turković, "Small-angle X-ray scattering and wide-angle $\mathrm{X}$-ray diffraction on thermally annealed nanostructured $\mathrm{TiO}_{2}$ films," Thin Solid Films, vol. 419, no. 1-2, pp. 105-113, 2002.

[17] H. Amenitsch, S. Bernstorff, and P. Laggner, "High-flux beamline for small-angle x-ray scattering at ELETTRA," Review of Scientific Instruments, vol. 66, no. 2, pp. 1624-1626, 1995.

[18] P. Dubček, "Nanostructure as seen by the SAXS," Vacuum, vol. 80, no. 1-3, pp. 92-97, 2005.

[19] B. D. Cullity and S. R. Stock, Elements of X-Ray Diffraction, Prentice Hall, New York, NY, USA, 2001.

[20] A. Guinier, "La diffraction des rayons $\mathrm{X}$ aux tres petits angles; application a l'etude de phenomenes ultramicroscopiques," Annals of Physics, vol. 12, pp. 161-237, 1939.

[21] G. Porod, "Die Röntgenkleinwinkelstreuung von dichtgepackten kolloiden Systemen-I. Teil," Kolloid-Zeitschrift, vol. 124, no. 2, pp. 83-114, 1951.

[22] C. S. Barret and T. B. Massalski, Structure of Metals, Pergamon Press, Oxford, UK, 1980. 

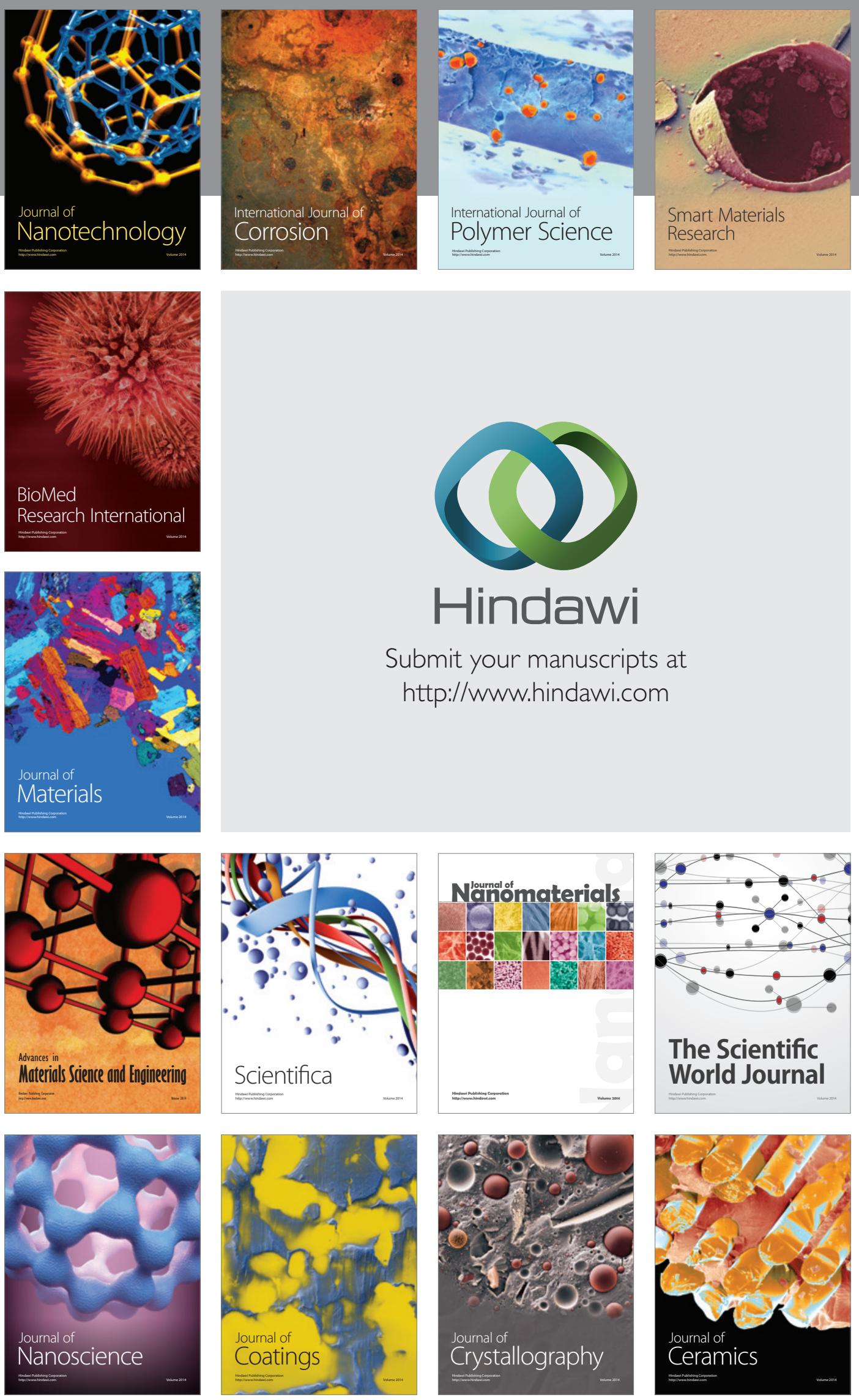

The Scientific World Journal

Submit your manuscripts at

http://www.hindawi.com

\section{World Journal}

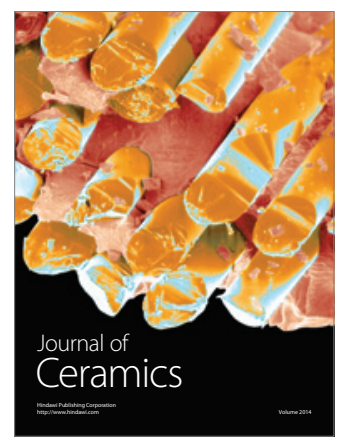

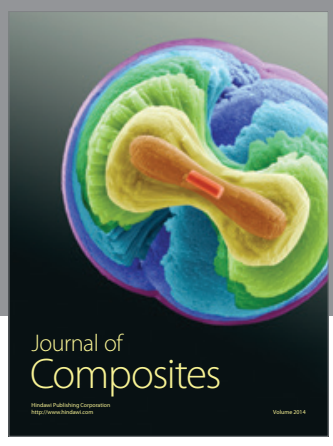
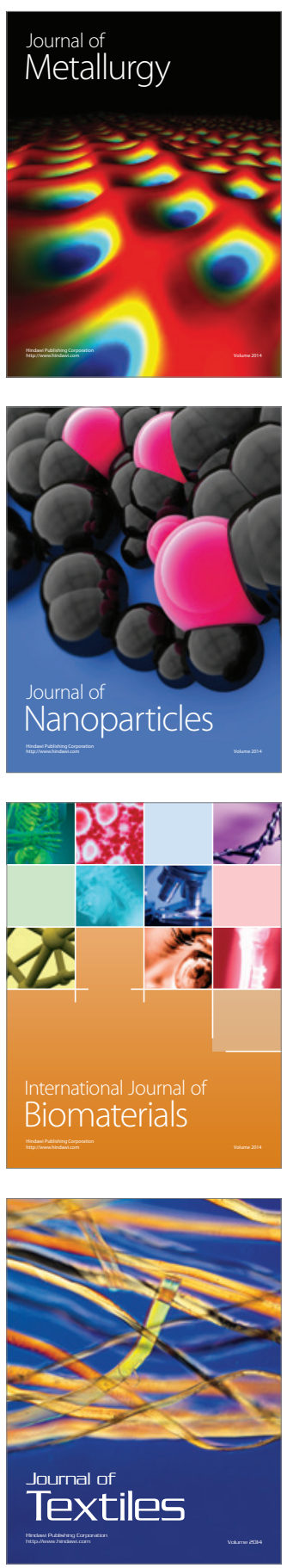